\title{
SOLITONS FROM SINE WAVES: ANALYTICAL AND NUMERICAL METHODS FOR NON-INTEGRABLE SOLITARY AND CNOIDAL WAVES
}

\author{
John P. BOYD \\ Department of Atmospheric and Oceanic Science, University of Michigan, 2455 Hayward Avenue, Ann Arbor, MI 48109, USA
}

Received 20 September 1984

Revised manuscript received 10 April 1986

\begin{abstract}
The "FKDV" equation, $u_{t}+u u_{x}-u_{x x x x x}=0$, is used as a testbed for a variety of analytical and numerical methods that can be applied to solitary waves and cnoidal waves of "non-integrable" differential equations, that is to say, to equations which cannot be solved by the inverse scattering transform. The basic tools are (i) Padé approximants formed from power series in the amplitude; (ii) a Newton-Kantorovich/pseudospectral Fourier/continuation numerical method; (iii) singular perturbation theory for two interacting solitons of almost identical phase speed; (iv) bifurcation and branch-switching methods; ( $v$ ) the imbricate-soliton series. A number of new results for the FKDV equation are obtained including extensive numerical calculations of the spatially periodic solutions with one peak ("cnoidal wave") and two peaks ("bicnoidal wave") per period, an analytical expression for the double-peaked soliton ("bion"), calculation of both the limit and bifurcation points for the bicnoidal wave, and finally the computation of accurate analytical approximations to the cnoidal wave for all amplitudes. More important, all of these analytical and numerical tools are highly effective for this equation in spite of the fact that it cannot be solved by the inverse scattering transform. Work now in progress will apply these methods to non-integrable equations in two space dimensions.
\end{abstract}

\section{Introduction}

The generalized Korteweg-deVries equation

$u_{t}+u u_{x}-u_{x x x x x}=0 \quad$ ["FKDV Eq.]

is a physical model (in the appropriate parameter range) for magneto-acoustic waves, gravitycapillary water waves, and waves in a nonlinear LC circuit with mutual inductance [13], but its greatest usefulness has been as a theoretical and numerical testbed for nonlinear waves. Although this equation, which we shall call the "Fifthdegree Korteweg-deVries" or "FKDV" for short, differs from the classical Korteweg-deVries only through the replacement of the third derivative by the fifth derivative, the different dispersion causes profound alterations in behavior. Unlike the KDV equation, the FKDV cannot be solved by the "inverse scattering" method; it is "non-integrable" in the jargon of wave theory, and it has a much richer phenomenology. In particular, the FKDV equation - but not the $\mathrm{KdV}$ - allows bound states of solitons ("bions"), chaos and loss of predictability, and the formation of solitary waves that oscillate in space or time or both. Fortunately, however, the FKDV equation has but a single space dimension and satisfies a similarity law (section 2) which makes it possible to investigate its solitary and cnoidal waves with great thoroughness. Because of these dual virtues of simplicity and rich phenomenology, researchers like Nagashima and Kuwahara [9], Imada [6], Yoshimura and Watanabe [13], and Gorshkov, Ostrovskii, and Papko [1-5] have previously studied this equation.

The philosophy of this work is similar: to employ the FKDV equation as a vehicle to illustrate and explore the usefulness of a variety of analytical and numerical methods for attacking solitary and cnoidal waves. A note on terminology: we shall use "soliton" as a synonym for "solitary 
wave" even though purists would restrict this term to solutions of "integrable" equations, and we shall similarly use "cnoidal" wave to describe the spatially periodic generalization of the soliton even though it is not generally possible to describe this wave in terms of the elliptic cosine function. We shall also restrict attention to steadily translating waves $-u(x, t)=u(x-c t)$ - on the spatially periodic domain $x \in[-\pi, \pi]$. The FKDV equation simplifies to the ordinary differential equation

$(u-c) u_{X}-u_{X X X X X}=0$,

where $c$ is the phase speed and $X \equiv x-c t$. When $c \approx-1, u(X) \approx a \cos (X)$ where the constant $a$ is small; as the amplitude increases, the wave becomes more and more narrow so that the solitary wave corresponds to the limit of an infinitely tall and infinitely narrow cnoidal wave. Section 2 is a briefing on the important properties of the solutions of (1.2).

The main tools discussed below are the following:

(i) Stokes' expansions/Padé approximants (section 3);

(ii) Newton-Kantorovich/pseudospectralFourier/continuation polyalgorithm and parameter exchange for limit points (section 4);

(iii) perturbation theory for two interacting solitons (section 5);

(iv) bifurcation and branch-switching methods (section 6);

(v) “imbricate" series (section 7).

The paper ends with a summary and prospectus. All the methods described above work well for this simple test problem, but the real payoffs will come in extending them to solitary waves in two and three spatial dimensions. Section 8 will discuss the possibilities and the problems.

\section{The fifth-degree Korteweg-deVries equation: background}

This section reviews some basic properties of the FKDV equation $[6-8,12,13,19]$. It also explains some conventions and definitions that will be used in the rest of the article.

Theorem 1. If $u(x, t)$ is a solution of the FKDV equation (1.1), then

(a) $v \equiv M+u(x-M t, t)$,

(b) $w \equiv c u\left(c^{1 / 4} x, c t\right)$,

are also solutions where $M$ and $c$ are arbitrary constants.

Proof. Direct substitution into (1.1); one finds that the constants $M$ and $c$ simply cancel out. Part (b) has been used by Kawahara [8] and Nagashima and Kuwahara [9].

In words, (a) states that one can always add a constant to a solitary or cnoidal wave provided that one simultaneously adds the same constant to the phase speed $c$. Because of this, the soliton and cnoidal wave are not uniquely specified until one adopts some convention. There are two obvious choices:

$$
\begin{array}{ll}
u( \pm \infty)=0 & (\text { if } X \in[-\infty, \infty]) \\
u( \pm \pi)=0 & (\text { if } X \in[-\pi, \pi]) \\
& \text { "soliton convention", }
\end{array}
$$

or

(ii) $\int_{-\pi}^{\pi} u(X, t) \mathrm{d} X=0$, "cnoidal convention",

where $X(\equiv x-c t)$ is the moving coordinate. The first choice, (2.3), is the most natural for solitary waves since it implies that the soliton asymptotes to 0 at infinity. The second choice, (2.4), requires that the constant in the Fourier cosine series for the cnoidal wave equal 0 . This is easy to impose by using a pseudospectral basis for $u(X)$ that includes only cosines and omits the constant. Most numerical results will be given in the "cnoidal convention", but it will sometimes be necessary to shift into the "soliton convention" to make accu- 
rate comparisons with previous solitary wave calculations.

The second part of the theorem, the "similarity law", is even more important because it implies that each soliton species can be described by a single, universal function containing no parameters. The universal function for the soliton-the soliton for unit phase speed - is given in analytic form, derived via curve-fitting, by Nagashima and Kuwahara [9]. The soliton for arbitrary height, width, or speed can then be obtained by rescaling the Nagashima-Kuwahara $f(X)$ according to (2.2). The two-peaked soliton or "bion" can be described by a similar universal function which is computed here for the first time.

For the cnoidal wave, the imposition of finite boundary conditions introduces another parameter so that we must compute a one-parameter family of solutions. The similarity law (2.2) then implies that we can choose any period we wish to compute this one-parameter family since cnoidal waves periodic with different periods can always be computed from those of period $2 \pi$ by rescaling.

It is obvious that a cnoidal wave with a period of $2 \pi / n$ where $n$ is an integer also satisfies the condition $u(-\pi)=u(\pi)$, so there is actually a countable infinity of cnoidal waves on the interval $X \in[-\pi, \pi]$ which can be labelled by $n$, the number of peaks on the interval. In the limit of infinitesimal amplitude, the phase speed and eigenfunction for each branch are $[c \approx-1, u \approx$ $\cos (X)], \quad[c \approx-16, u \approx \cos (2 X)], \ldots\left[c \approx-n^{4}, u\right.$ $\approx \cos (n X)]$, etc. To understand the ordinary cnoidal wave (which tends to the single-peaked soliton in the limit of large amplitude), it suffices to compute only one of this infinity of discrete branches, and we will normally pick $n=1$ - one peak on each spatial interval. However, the bion and the corresponding bicnoidal wave emerge as subharmonic bifurcations of the $n=2$ cnoidal wave (section 6), so it is necessary to consider this branch-which is just the rescaling of the $n=1$ solution - in order to understand the bifurcation.

The final preliminary issue is this: how do we define the border between the soliton and sine wave regimes? For very large amplitude, the peaks of the cnoidal wave are narrow and overlap by only an exponentially small amount, so it is a good approximation to represent the wave as an "imbricate" series of solitons, one soliton centered at $X=0, \pm 2 \pi, \pm 4 \pi, \ldots$ as in (7.2). (See section 7 for further discussion). For very small amplitude, nonlinear effects are weak and the cnoidal wave is well approximated by an ordinary sinusoidal wave, which is the starting point for the "Stokes' expansion" of section 3 .

Fig. 1 is a graph of the exact cnoidal wave for $a=10$ and the soliton and sine wave approximations to it. Note two conventions that will be used throughout the rest of this paper: (i) the phase of the wave is chosen so that it is symmetric about $X=0$, so only half the interval need be graphed; and (ii) the amplitude $a$ is defined to be the coefficient of $\cos (X)$ in the Fourier expansion of the wave, not the difference between the maximum and minimum of $u(X)$. (This definition of "amplitude" simplifies analysis of the Stokes' expansion and the exact numerical solutions discussed below.)

The graph shows, as similarly found by Boyd [21] for the Korteweg-deVries equation, that there

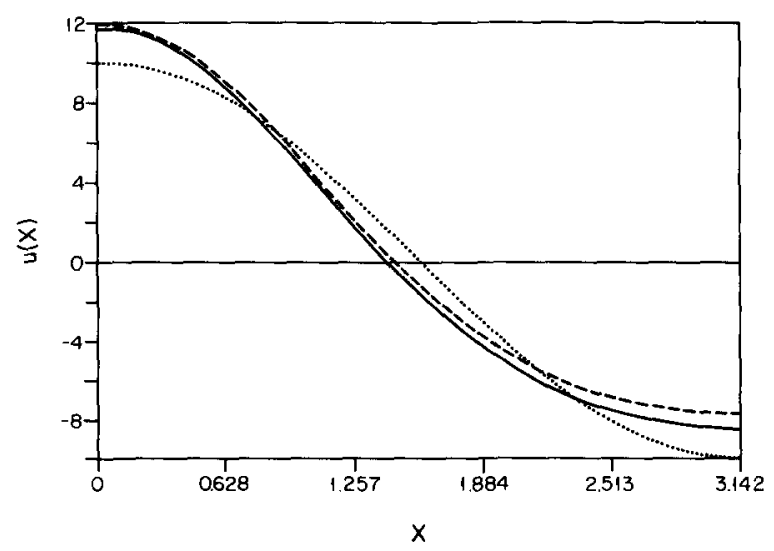

Fig. 1. The cnoidal wave for $a=10$ [solid line] is compared with $v(X) \equiv a \cos (X)$ [dotted line] and with $w(X) \equiv$ $c f\left[c^{1 / 4} X\right]-c$ [dashes] where $f(X)$ is the soliton for unit phase speed and $c=7.181$ is the phase speed in the "soliton" convention. Note that because all the waves (and approximations) are symmetric about $X=0$, only the positive half of the interval $X \in[-\pi, \pi]$ is shown in this graph and the figures below. 
is a strong overlap between the sine wave and soliton regimes: both lowest order approximations agree well with the exact solution for $a=10$. It follows that for intermediate $a$, the cnoidal wave can be legitimately regarded as either a soliton or a sine wave, whichever is more convenient.

\section{Stokes' expansions and Padé approximants}

When the amplitude is very small so that the nonlinearity can be neglected, the FKDV equation (1.2) has the approximate solution

$u_{1}(X) \approx a \cos (X) ; \quad c_{0} \approx-1$.

In 1847, Sir G.G. Stokes showed that this approximation can be improved by expanding both $u(X)$ and the phase speed $c$ in powers of the amplitude $a$ where "amplitude" in this context means "the coefficient of $\cos (X)$ ". Defining

$u(X) \equiv \sum_{i=1}^{N} u_{i}(X) a^{i} ; \quad c \equiv \sum_{i=0}^{N-1} c_{i} a^{i}$

where $N$ is the highest order to which the perturbation expansion will be carried out, matching powers gives at each order

$$
\begin{gathered}
-u_{i, X X X X X}-c_{0} u_{i, X}=f_{i}(X), \\
f_{i}(X)=\sum_{k=1}^{i-1} c_{i-k} u_{k, X}(X)-\frac{1}{2}(\mathrm{~d} / \mathrm{d} X) \\
\quad \times\left[u_{k}(X) u_{i-k}(X)\right] .
\end{gathered}
$$

This same device is known by a variety of other names including the "Poincaré-Lindstedt" technique, the "method of strained coordinates", and so on [10].

By induction, one can prove that the exact solution to (3.3) is a finite Fourier cosine series:

$$
u_{i}(X)=\sum_{j=1}^{i} u_{i j} \cos (j X)
$$

Note that the cosine functions automatically and individually satisfy the boundary conditions of periodicity with period $2 \pi$; it is only necessary to compute the coefficients of the Fourier expansion of $f_{i}(x)$,

$$
f_{i}(X)=\sum_{j=1}^{i} f_{i j} \sin (j X)
$$

and then

$u_{i j}=f_{i j} /\left[j^{5}-j\right]$.

Eq. (3.7) has no bounded solution unless $f_{i 1}=0$ for all $i$ because the denominator in (3.7) is infinite for $j=1$. This reflects the fact that $\sin (X)$ is a homogeneous solution of (3.3), so the equation is not solvable unless the forcing is orthogonal to $\sin (X)$. Fortunately, the phase speed to this order, $c_{i-1}$, is still undetermined. (Note from (3.1) and (3.2) that the expansion for $c$ starts with one lower power of $a$ than that for $u(x)$ so that at the $i$ th order, the unknowns are always $u_{i}$ and $c_{i-1}$ ). If we split the forcing into a part which is proportional to the phase speed correction and a part which is not, i.e.

$f_{i}(x)=F_{i}(x)+c_{i-1} u_{1, X}$,

then since $u_{1, X}=\sin (X)$, we can eliminate the otherwise unbounded $j=1$ term in (3.6) by imposing

$c_{i-1}=-F_{i 1} \quad$ ["solvability condition"],

where $F_{i 1}$ is the coefficient of $\sin (X)$ in $F_{i}(x)$.

The nonlinear terms in (3.4) can be easily converted from products of cosines into a Fourier series by using the familiar identity

$$
\begin{aligned}
& \cos (m X) \cos (n X)=\frac{1}{2}\{\cos ([m+n] X) \\
& \quad+\cos (|m-n| X)\} .
\end{aligned}
$$

The one modest complication is that the absolute value in (3.10) makes it rather messy and confus- 
ing to try to express the Fourier coefficients of $F_{i}(x)$ as an explicit formula. However, it is easy to describe them in terms of a computational procedure.

As a check, the series were first computed to low order and verified using the algebraic manipulation language REDUCE by direct resubstitution of the series in the original differential equation. Table I gives the calculated series.

Since REDUCE is roughly 100 to 1000 times slower than a numerical (as opposed to symbolic) calculation, the series was recomputed to higher order using BASIC. The 23 line program required less than 3 minutes on an IBM PC, even when run in interpreted mode, to calculate the Stokes' expansion to 11th order [10th order in $c$ ]. The motive for using the most humble of languages on the most humble of microcomputers is to emphasize that Stokes' method is extremely simple and fast - and can easily be extended to compute cnoidal waves in two or more spatial dimensions.

The operation count is $\mathcal{O}\left(N^{4}\right)$ where $N$ is the highest perturbation order computed. At each order, we have a loop over $k$, the sum index in (3.4); to evaluate the nonlinear term, we must trace loops in the indices $m$ and $n$, which mark the Fourier components of each of the two factors of $u(x)$. Because the array of Fourier coefficients is lower triangular, i.e.

$u_{i j}=0 \quad$ if $i<j$,

the indices $m$ and $n$ have upper bounds of $i$, so the work at each perturbation order is at most $\mathcal{O}\left(i^{3}\right)$, and when $i$ varies from 2 to $N$, the number of operations is therefore at most $\mathcal{O}\left(N^{4}\right)$. The operation count can be reduced to only $\mathcal{O}\left(N^{3} \log _{2} N\right)$ by using the fast Fourier transform [20] to compute the Fourier series of the nonlinear terms.

Naively, one might suppose that in two space dimensions, a similar expansion would require $\mathcal{O}\left(N^{6}\right)$ operations since the Fourier indices $m$ and $n$ must become four indices. However, by careful accumulation of partial sums as explained in
Orszag [20], the operation count can be reduced to $\mathcal{O}\left(N^{5}\right)$ in two space dimensions and $\mathcal{O}\left(N^{6}\right)$ in three space dimensions even without the fast Fourier transform.

Thus, Stokes' method-even if high order $N$ is desired - is applicable to multi-dimensional problems at modest cost. The major problem is that the perturbation series has only a finite radius of convergence. Fig. 2 compares the $N=11$ approximation for $c$ with the exact phase speed. Agreement is good for $a<30$, but the approximation then rapidly diverges with higher $a$ and is already double the true phase speed for $a=40$.

Fig. 1 has already shown, however, that even for $a$ as small as 10 , the cnoidal wave is closely approximated by a sum of evenly spaced solitons. Thus, the Stokes' series has indeed generated "solitons from sine waves" as promised in the title of the paper.

This broad range of usefulness can be greatly extended via Padé approximants [14]. The $[K, L]$ approximant is a rational function whose numerator is degree $K$ with denominator of degree $L$. Without loss of generality, the constant in the denominator polynomial can be set equal to 1 ;

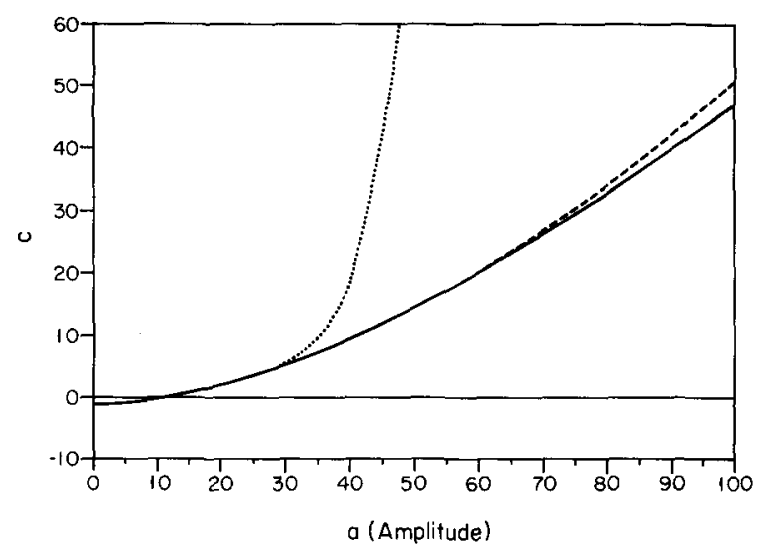

Fig. 2. Phase speed $c$ for the cnoidal wave as a function of amplitude $a$. The exact $c$ is shown as the solid line, the Stokes' approximation through $N=10$ is the dotted curve, and the [3, 2] Padé approximant formed from the Stokes' series is dashed. The two approximations are graphically indistinguishable from the exact $c$ for $a<30$, but the Stokes' expansion is off the graph for $a>45$. The Padé approximant remains close to the true speed even for $a$ as large as 100 . 
Table I

Stokes expansions for the phase speed $c$ (in the "cnoidal convention" (2.3)) and for Fourier cosine coefficients $a_{j}$ where $u(X)=\sum a_{j} \cos (j X)$. For brevity, results are listed only through 7 th order (6th order in $c$ ) although values through $N=11$ were used to generate figs. 1-3.

\begin{tabular}{lllllllll}
\hline & $a^{0}$ & $a^{1}$ & $a^{2}$ & $a^{3}$ & $a^{4}$ & $a^{5}$ & $a^{6}$ & $a^{7}$ \\
\hline$c$ & -1 & 0 & 0.0083333 & 0 & $-2.0255 \mathrm{E}-6$ & 0 & $1.4875 \mathrm{E}-9$ & 0 \\
$a_{2}-$ & - & 0.016666 & 0 & $-5.7870 \mathrm{E}-6$ & 0 & $4.2623 \mathrm{E}-9$ & 0 \\
$a_{3}-$ & - & - & $1.0416 \mathrm{E}-4$ & 0 & $-4.4041 \mathrm{E}-8$ & 0 & $3.2251 \mathrm{E}-11$ \\
$a_{4}-$ & - & - & - & $4.7658 \mathrm{E}-7$ & 0 & $-2.8757 \mathrm{E}-10$ & 0 \\
$a_{5}-$ & - & - & - & - & $1.7730 \mathrm{E}-9$ & 0 & $-1.3206 \mathrm{E}-12$ \\
$a_{6}-$ & - & - & - & - & - & $5.8461 \mathrm{E}-12$ & 0 \\
$a_{7}-$ & - & - & - & - & - & - & $1.7717 \mathrm{E}-14$ \\
\hline
\end{tabular}

this leaves $(K+L+1)$ undetermined coefficients which are chosen so that the Taylor series of the $[K, L]$ approximant agrees with the Stokes' expansion through the first $(K+L+1)$ terms. The Padé approximants of all orders can be calculated by solving sets of linear algebraic equations. Although the convergence theory for Padé approximants has gaps, numerical experience and theoretical arguments suggest that if $f(a)$ is well-behaved for all real $a$, its Padé approximant will converge along the whole real axis even if the power series from which it is formed has only a finite radius of convergence.

Table I shows that the amplitude expansions for $c$ and various Fourier coefficients of $u(X)$ are in powers of the square of $a$ rather than $a$ itself, so we shall take $y=a^{2}$ as the variable in computing Padé approximants for $c$ and each Fourier coefficient. We also note that the coefficient of $\cos (j X)$ is $\mathcal{O}\left(a^{j}\right)$, so-after factoring out $a^{j}$-we must resign ourselves to lower and lower order Padé approximants as $j$ increases. With $N=11$, we have a maximum of six terms (including the constant) for $c$, so the highest approximant we can form is the $[3,2]$ in $a^{2}$. We can form $[2,2]$ approximants for the coefficients of $\cos (2 X)$ and $\cos (3 X),[1,1]$ approximants for the coefficients of $\cos (4 X)$ and $\cos (5 X)$, and no useful approximants for the higher Fourier terms, which we simply drop.

Nonetheless, fig. 2 shows that the [3, 2] Padé approximant to $c$ has triple the range of accuracy of the $N=11$ perturbation series. We chose the [3, 2] approximant rather than some other because one can show from the similarity law (2.1) that $c \sim \mathcal{O}\left(a^{4 / 3}\right)$ for large $a$ : the [3,2] approximant grows too fast $\left[\mathcal{O}\left(a^{2}\right)\right]$ while the $[2,2]$ grows too slowly. In point of fact, one finds that these two approximants tend to bracket the correct answer, which is lower than the $[3,2]$ value by about $1 / 3$ the difference between the $[3,2]$ and $[2,2]$ approximants.

Figs. 3 and 4 illustrate the exact and approximate $u(X)$ where the latter used Padé approxi-

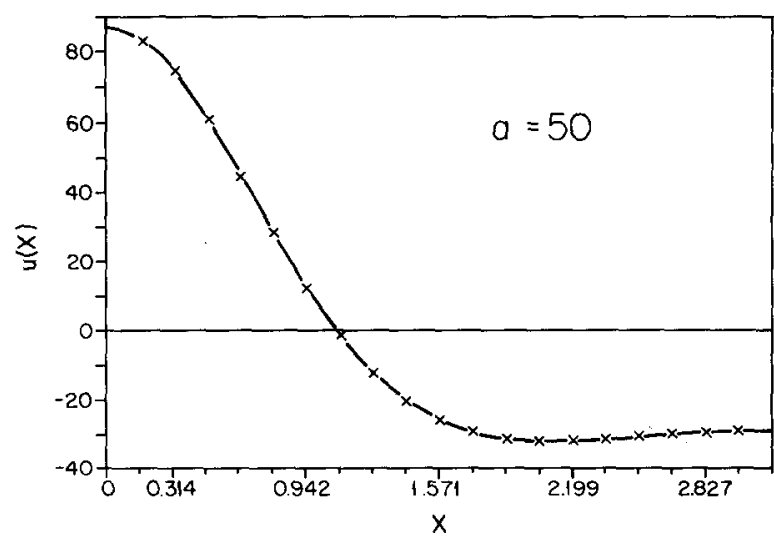

Fig. 3. Comparison of the exact cnoidal wave $u(X)$ with the best Padé approximant to it for $a=50$. The mathematical form of the Padé approximant is given in the text, but it was constructed solely from the terms through $N=11$ in the Stokes' expansion. The exact solution is shown as a solid line and the approximation as a dashed line with crosses-but because the two curves agree to within the thickness of the curve, only the crosses are visible. 


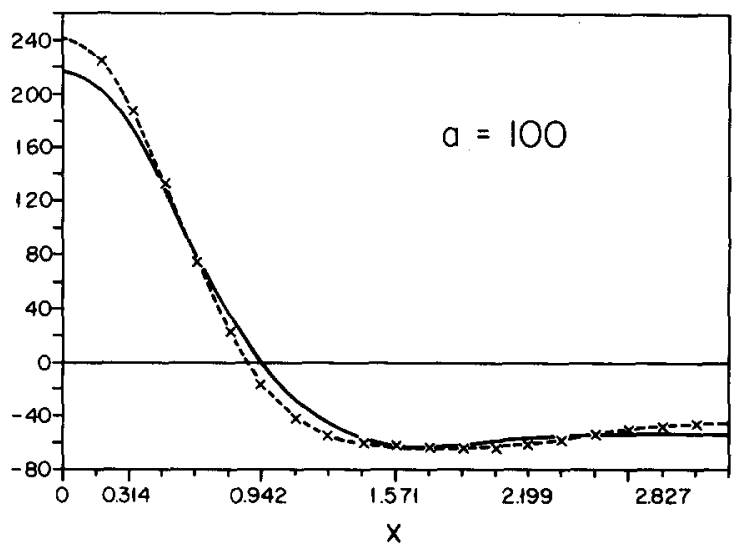

Fig. 4. Comparison of the exact cnoidal wave with the best Padé approximant for $a=100$. As in fig. 4 , the exact wave is the solid curve and the approximation is a dashed line with crosses.

mants for the coefficients of $\cos (2 X)$ through $\cos (5 X)$ as explained above. For $a=50$ - outside the radius of convergence of the unmodified power series - the Padé approximation to $u(X)$ is indistinguishable from the exact solution to within the thickness of the curve. For $a=100$, the error is noticeable but still small. As noted in Section 2, even $a=10$ is nearer to the pure soliton than to the linear cosine wave, so the success shown in fig. 4 for an amplitude 10 times larger is rather impressive.

Thus, the conclusion is that Stokes' series - even without conversion into Padé approximants - is not merely a small amplitude expansion (though this is how it is formally justified). Rather, the Stokes'-Padé approximants are a powerful way to extrapolate from the linear, small amplitude regime well into the soliton regime, justifying the title of this article: "Solitons from sine waves".

\section{The Newton-Kantorovich/pseudospectral Fourier/continuation polyalgorithm}

\subsection{Newton-Kantorovich method}

Despite this section's formidable title, it is actually quite easy to numerically compute the cnoidal and solitary waves, and the three algorithms that are used are all familiar and well-tested ideas. Our problem is

$-u_{X X X X X}-c u_{X}+u u_{X}=0$,

$u(\pi)=u(-\pi)$

Because this is nonlinear, the phase speed $c$ is not a discrete eigenvalue, as for a linear wave problem, but rather is a continuous parameter. As $c$ is varied, the wave will adjust its amplitude accordingly.

As noted by Norton [24] and earlier and more abstractly by Kantorovich [25], one can apply Newton's method directly to a nonlinear differential equation to reduce it to a sequence of linear differential equations. If we now write

$u=u_{k}(X)+\Delta(X)$

and define the linear operator $L$ via, for any $\Delta$,

$L \Delta \equiv-\Delta_{X X X X X}-c \Delta_{X}+u_{k} \Delta_{X}+u_{k, X} \Delta$,

then direct substitution into (4.1) gives, without approximation,

$L \Delta=-L u_{k}-\Delta \Delta_{X}+u_{k} u_{k, X}$

If $u_{k}(X)$ is a known approximate solution to (4.1) so that $\Delta(X) \ll 1$, we can neglect the quadratic $\Delta \Delta_{X}$ term in (4.4) so that it becomes a linear equation to compute the correction $\Delta(X)$. Each Newton-Kantorovich iteration consists of solving

$L \Delta_{k}=-L u_{k}+u_{k} u_{k, X}$

followed by the replacement of $u_{k}(X)$ by the improved guess

$u_{k+1}=u_{k}+\Delta_{k}$.

Since this iteration, like Newton's method for solving an algebraic equation, is based on linearization, it is usually called the "NewtonKantorovich" method. The name of the Russian 
mathematician is inciuded in the title of the algorithm as a reminder that we are using the Taylor expansion of a functional rather than a function, and $\Delta$ is not merely a numerical correction, but a function of $X$.

There are two technical problems: (i) obtaining a sufficiently good first guess for $u(X)$ so that the iteration converges, which will be done via the continuation method described in the third subsection below; and (ii) zero eigenvalues of the operator $L$. As the parameter $c$ is varied, the eigenvalues of $L$ will vary, too, and one or more may cross zero for a particular value of $c$. These isolated zero eigenvalues are either limit points, explained in subsection (iv) below, or bifurcation points, which are discussed in section 6 .

In addition, $L$ has two eigenfunctions with zero eigenvalue for any $c$ because of continuous symmetries of (4.1) and (4.2). The first is translational invariance: if $u(X ; c)$ is a solution, then so is $u(X+\phi ; c)$ for any constant $\phi$. This implies that $e_{1}(X) \equiv u_{X}$ is a eigenfunction of $L$ with zero eigenvalue. We can suppress translational freedom by imposing $\mathrm{d} u / \mathrm{d} X=0$ at $X=0$. For this particular problem, the soliton and cnoidal wave are symmetric about their maxima, so an equivalent alternative is to impose symmetry with respect to $X=0$ on the numerical solution.

The second is a consequence of theorem 1a of section 2: because it is possible to alter the phase speed of a solution by simultaneously adding a constant to both $c$ and $u(X), L$ must also have the eigenfunction

$e_{2}(X) \equiv-1+\partial u(X ; c) / \partial c$

where $u(X ; c)$ is the cnoidal wave. We can suppress this linear eigenfunction by applying either of the two conventions (2.3) or (2.4).

\subsection{Fourier pseudospectral method}

Since the boundary conditions are periodic, the most efficient way to solve (4.6) is by expanding the solution as a Fourier series,

$\Delta(X)=\sum_{n=1}^{N} d_{j} \cos (j X)$,

where $N$ is the chosen truncation. This numerical method also makes it easy to impose the conditions that eliminate the two null eigenvalues of $L$. By including only cosines in the basis, we force the numerical solution to be symmetric about the origin: this suppresses the translational degree of freedom. Eliminating the constant from the Fourier series imposes the "cnoidal convention" (2.4). With a slight change of basis, the same combination of algorithms can be applied to compute the soliton on $X \in[-\infty, \infty]$ as explained in Boyd [17].

The "pseudospectral" method, also known as "orthogonal collocation", obtains a set of linear algebraic equations to determine the $d_{j}$ by substituting the Fourier series into the differential equation and then demanding that the differential equation be exactly satisfied at each of the $N$ "collocation" or "interpolation" points

$X_{i} \equiv \pi(2 i-1) /(2 N), \quad i=1,2, \ldots, N$.

The justification for the pseudospectral method is given in Boyd [15] and the monograph by Gottlieb and Orszag [16]; here, we will merely note that the algorithm gives roughly the same accuracy as the older Galerkin's method (unless $N \leq 3$ ) but is much easier to program and saves an extra computational loop. Thus, at each pass of the Newton's iteration, we must solve the linear matrix equation

$A d=f$,

where the matrix elements are given by

$$
\begin{aligned}
A_{i j} \equiv & \left.L \cos (j X)\right|_{X=X_{i}} \\
= & \left\{j^{5}-j\left[u_{k}\left(X_{i}\right)-c\right]\right\} \sin \left(j X_{i}\right) \\
& +u_{k, X}\left(X_{i}\right) \cos \left(j X_{i}\right), \\
f_{i} \equiv- & \left\{-u_{k, X X X X X}\left(X_{i}\right)\right. \\
& \left.+\left[u_{k}\left(X_{i}\right)-c\right] u_{k, X}\left(X_{i}\right)\right\}
\end{aligned}
$$


Table II

The Fourier cosine coefficients $a_{j}$ of the cnoidal wave for $a=100$

\begin{tabular}{lc}
\hline$j$ & $a_{j}$ \\
\hline 1 & 100.0 \\
2 & 70.85 \\
3 & 32.17 \\
4 & 9.913 \\
5 & 2.479 \\
6 & 0.5511 \\
7 & 0.1128 \\
8 & 0.0218 \\
9 & 0.0043 \\
10 & 0.0007 \\
\hline
\end{tabular}

and where the elements of the column vector $d$ in (4.11) are the Fourier cosine coefficients of the correction $\Delta(X)$. Given a good first guess for the Fourier coefficients, one must merely solve (4.9) repeatedly-an $N \times N$ matrix equation at each iteration - until the norm of $d$ is sufficiently small.

Table II shows the Fourier coefficients of $u(X)$ for $a=100$, which is far into the soliton regime as can be seen from fig. 4. As explained in [15] and [16], the geometric decrease of the Fourier coeffcients with $j$ is a general property of pseudospectral solutions. One can prove that for a smooth function, the error will decrease exponentially fast with $N$. Taking $N=6$ gave an accuracy of $1 \%$ or better for all $a \leq 100$.

\subsection{Continuation method}

There are still two remaining difficulties, alas. The first is inherent in the phrase "given a good first guess"; like any Newton iteration, (4.9) will not converge unless the initial iterate is reasonably close to the true solution. The remedy is the so-called "continuation" method. The idea is to begin with a known solution, which in this case is the limit of very small amplitude, $u(X) \approx a \cos (X)$ and $c \approx-1$, and use this as a first guess for $c$ close to -1 . After the solution has been obtained for (let us say) $c=-0.9$, one can use $u(X ; c=$ $-0.9)$ as a first guess for $u(X ; c=-0.8)$, which

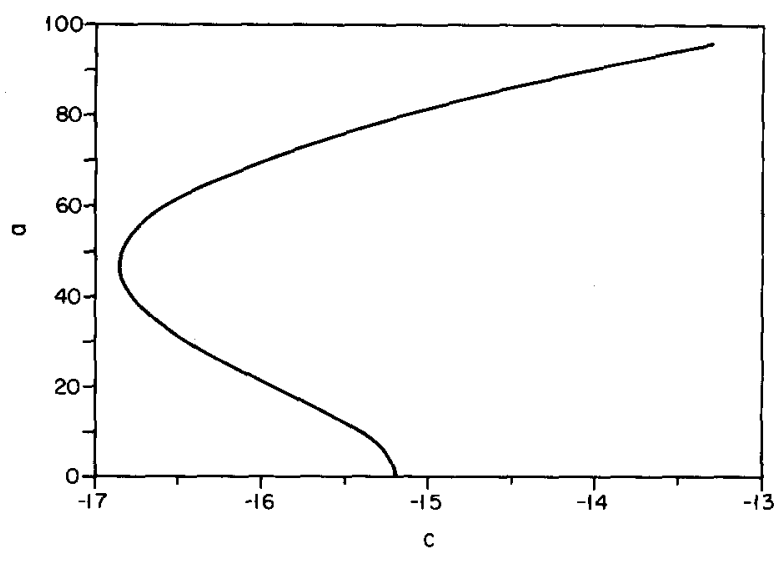

Fig. 5. Amplitude $a$ (defined to be the coefficient of $\cos (X)$ in the Fourier expansion of $u(X)$ ) for the bicnoidal wave as a function of phase speed. The graph for negative $a$ [not shown] is the reflection of the graph for positive $a$ with respect to the $c$-axis.

provides a first guess for $c=-0.7$, and so it goes. (One can equally well march in the amplitude $a$, which we define to be the coefficient of $\cos (X)$, and take $c$ as one of the unknowns, but the exposition is easier if we assume that we are marching in small steps in the phase speed $c$ ). Once two points have been computed, better first guesses can be obtained by the linear extrapolation

$a_{j}\left(c_{0}+\delta c\right) \approx a_{j}\left(c_{0}\right)+\left[a_{j}\left(c_{0}\right)-a_{j}\left(c_{0}-\delta c\right)\right]$,

where $\delta c$ is the step size; some researchers have used more sophisticated extrapolation schemes. There is no rigorous theory for picking the step size, but common sense works fairly well: if the iteration diverges, try again with smaller $\delta c$; if the solution for the next value of $c$ is very close to that just computed, try a larger stepsize in $c$.

\subsection{Pseudo-arclength continuation, limit points and parameter exchange}

Fig. 5 illustrates the Fourier coefficient $a_{1}$ as a function of the phase speed $c$ for the bicnoidal 
wave. When $c$ is the continuation parameter, a limit point is defined to be a place such that

$\partial u / \partial c \rightarrow \infty \quad$ as $c \rightarrow c_{\text {limit }}$.

Equivalently, it is a point where two branches of the solution smoothly merge. The bicnoidal wave has limit points at $c=-15.195$ and $c=-16.88$.

The standard remedy for a limit point is the "pseudo-arclength continuation" method developed by H. B. Keller and his collaborators [18]. The marching parameter becomes an approximation to the arclength $s$ and the size of the matrix system is increased by one because $c$ is now computed as a function of arclength along with all the Fourier coefficients. Keller's technique smoothly turned the corner at $c=-16.88$ and it was not necessary to decrease the step size in pseudo-arclength as the limit point was approached.

We omit a detailed discussion here because (i) the theoretical background is thoroughly explained in [18] and other articles it references and (ii) it is unnecessary for this problem. If we look at Fig. 5 carefully, we note that although $a(c)$ is double-valued, the phase speed is a single-valued function of the amplitude $a$. It follows that we can avoid limit points altogether by the method of "parameter exchange", that is, taking $a$ (三 the first Fourier coefficient, $a_{1}$ ) as the known parameter and solving for the phase speed $c$ and for the higher Fourier coefficients of $u(X)$ as the unknowns.

To apply the Newton-Kantorovich iteration, we write

$c=c_{k}+\delta c_{k}$,

redefine the correction $\Delta(x)$ in (4.3) to be

$\Delta(X) \equiv \sum_{j=2}^{N} d_{j} \cos (j X)$

which differs from (4.9) only in the lower index of summation (now 2 instead of 1 ) and redefine the operator $L$ in (4.4) by replacing $c$ by $c_{k}$. The iteration becomes

$L \Delta_{k}-\delta c_{k} u_{k, X}=-L u_{k}+u_{k} u_{k, X}$

Applying the standard pseudospectral condition that (4.19) be satisfied at the $N$ interpolation points given by (4.10) converts it into an $N \times N$ matrix problem for the unknowns.

All the calculations reported in this article were done in interpreted BASIC on an IBM PC in less than an hour of CPU time. Again, the point of using a slow microcomputer and an inefficient language is to emphasize that the four algorithms combined here are cheap and efficient. What can be done so quickly on a little computer in one dimension will be an inexpensive calculation on a "real" computer even in two dimensions.

\section{Solitons as particles: the Gorshkov- Ostrovskii-Papko theory of the bion}

We have seen in section 3 that it is easy to construct a perturbation theory for nonlinear waves for small amplitude, but it is very difficult to obtain any analytical results when the amplitude is large and nonlinear effects must be somehow included even in the zeroth-order approximation. The Russian scientists Gorshkov, Ostrovskii, and Papko [1-5], however, have shown and discussed one exception: a perturbation theory for interacting solitary waves that are travelling at approximately the same speed.

One inspiration for their study was P. Lax's [26] investigation of the collision of two solitons of the Korteweg-deVries equation. Lax showed that when the ratio of soliton velocities is less than 2.618 , the two solitons approach one another and exchange roles - the leading soliton, which is the smaller of the two before the collision, grows in amplitude during the interaction and is the larger as $t \rightarrow \infty$-but the two peaks separate without merging. The distance between the peaks is never less than some minimum separation. When the 
relative velocity of the two solitons is sufficiently small, the peaks remain so far apart that the mutual interaction of the two solitons remains small in comparison to their self-interaction at all times - and thus can be treated as a tiny perturbation to a pair of independent, isolated, noninteracting solitons. The key assumption is that

$c_{1}-c_{2} \ll 1$.

The most dramatic effect of the soliton collision is the exchange of energy between the two pulses. It is true for both the ordinary Kortweg-deVries equation and the FKDV equation that the phase velocity of a soliton completely determines its width and amplitude. Consequently, the paramount goal is to determine how $c_{1}$ and $c_{2}$ vary with time. As long as the two solitons remain far apart (as guaranteed by (5.1)), the shapes of the two peaks will closely resemble those of Korteweg-deVries or FKDV solitons with the phase speed determining all other quantities.

The slow variations of $c_{1}$ and $c_{2}$ with time can be found by applying a singular perturbation technique known as the "method of multiple scales" [10]. We assume that $c_{1}$ and $c_{2}$, instead of being constants, are functions of the "slow" time variable $\tau$ where

$\tau=\epsilon t$

where $\epsilon \ll 1$ is some nondimensional measure of the mutual interaction of the two solitons. Defining $f(X)$ to represent the FKDV soliton for unit phase speed in the soliton convention, we further assume

$$
\begin{aligned}
& u(x, t) \approx U_{1}\left(X_{1}\right)+U_{2}\left(X_{2}\right) \\
& +\epsilon\left[\Delta_{1}\left(X_{1}\right)+\Delta_{2}\left(X_{2}\right)+q(x, t)\right] \text {, } \\
& U_{i} \equiv c_{i}(\tau) f\left[c_{i}(\tau)^{1 / 4} X_{i}\right], \quad i=1,2, \\
& X_{i} \equiv x-\int^{\tau} c_{i}\left(\tau^{\prime}\right) \mathrm{d} \tau^{\prime}, \quad i=1,2 .
\end{aligned}
$$

The integrals over $\tau$ in (5.5) allow for the accumulation of phase variations as $c_{1}$ and $c_{2}$ vary with time; they are analogous to the phase integrals of the WKB approximation.

Let us now focus upon the neighborhood of the soliton labeled " 1 ". At zeroth order, we see from (5.3) that

$-U_{1, X X X X X}-c_{1} U_{1, X}+U_{1} U_{1, X}=0$,

where we have written $X$ as a shorthand for $X_{1}$, which implies that to zeroth order, each soliton is isolated and independent, and the slow time variable $\tau$ appears only as a parameter.

At first order in $\epsilon$, we have

$$
-\Delta_{1, X X X X X}-c_{1} \Delta_{1, X}+\left[U_{1} \Delta_{1}\right]_{X}=H(X),
$$

where

$$
H(X)=-\left\{U_{1, \tau}+\left[U_{1} U_{2}\right]_{X}\right\}
$$

It is only at this order that the slow time derivative appears, and then only operating on the zeroth order solution. The solvability condition for (5.7) will in fact determine how $U_{1}$ and therefore $c_{1}$ varies with $\tau$. However, before we proceed to compute this $\tau$-dependence, we need to discuss some additional approximations that are implicit in (5.7)-(5.8).

The first is that we assume that everything in the neighborhood of the first soliton is a function only of $X_{1}$; we again omit the subscript in writing (5.7) and (5.8). In reality, it is known [12] that two colliding FKDV solitons lose a little energy to a dispersive wave train - the term $q(x, t)$ in (5.3) and therefore are a bit smaller after the collision than before. When the solitons always remain far apart, however, this "scattering" or "dispersive" loss is small in comparison to the amplitude of the solitons, and thus can be legitimately neglected to lowest order.

The second problem is that $U_{2}$ is actually a function of $X_{2}$ rather than $X_{1}$. However, since 
$c_{1} \approx c_{2}$,

$X_{2}=X_{1}-s(\tau)+\mathcal{O}(\epsilon)$,

where $s(\tau)$ is the separation between the two solitons. Thus,

$$
\begin{aligned}
& U_{1}\left(X_{1}\right) U_{2}\left(X_{2}\right)=U_{1}\left(X_{1}\right) \\
& \quad \times U_{2}\left(X_{1}-s[\tau]\right)[1+\mathcal{O}(\epsilon)]
\end{aligned}
$$

and the approximation is uniform with time provided that we allow the separation $s(\tau)$ to vary slowly with time. Another way of putting it is that we can legitimately replace $c_{1}$ and $c_{2}$ by a common value $c$ everywhere except where these phase speeds are differentiated.

The solvability condition for (5.7) is that the inhomogeneous term, $H(X)$, must be orthogonal to the discrete eigensolutions of the adjoint of the linear operator on the left-hand side of the equation. McLaughlin and Scott [22] and Keener and McLaughlin [23] exploit similar conditions to obtain ordinary differential equations in time to describe the slow variations of sine-Gordon solitons. We can understand the need for this condition by recalling that the adjoint of an operator $L$ is that operator $L^{*}$ such that for any functions $v$ and $w$ which satisfy the specified boundary conditions,

$\langle v, L w\rangle=\left\langle L^{*} v, w\right\rangle$

where

$\langle a, b\rangle \equiv \int_{-\infty}^{\infty} a(X) b(X) \mathrm{d} X$

for any functions $a(X)$ and $b(X)$ and the boundary conditions are that $v(X)$ and $w(X)$ decay exponentially as $|X| \rightarrow \infty$. Repeated integration-by-parts of (5.11) shows that

$$
L^{*} v \equiv-\left\{-v_{X X X X X}-c_{1} v_{X}+U_{1} v_{X}\right\}=0 .
$$

Comparing (5.13) with (5.6), we see that $U_{1}(X)$ is in fact a discrete solution of $L^{*} v=0$. Thus, we must have

$$
\left\langle U_{1}, H\right\rangle=0 \quad \text { ["solvability condition"] }
$$

for (5.7) to have a bounded solution. The necessity for (5.14) follows trivially from (5.11), the definition of the adjoint: if we let $v=U_{1}$ and $w=\Delta_{1}$, then $L^{*} U_{1}=0$ implies $\left\langle L^{*} U_{1}, w\right\rangle=0$, which means $\left\langle U_{1}, L w\right\rangle=\left\langle U_{1}, H\right\rangle=0$, which is just (5.14).

By integrating the interaction term by parts (twice), we can rewrite (5.14) as

$$
\left\langle U^{2}\right\rangle_{\tau}=-\left\langle U(X)^{2}[U(X-s)]_{X}\right\rangle,
$$

where, since $c_{1} \approx c_{2}$, we have replaced $U_{2}(X-s)$ by $U_{1}(X-s)$, and then dropped the subscripts. Eq. (5.15) is the "holy grail" of the perturbation theory since it gives the slow time dependence induced in the solitons due to their mutual interaction; more general perturbations can be handled along the same lines, as in [22] and [23], by replacing the r.h.s. by something different. (Eq. (6) of [1] is identical except for a typographical error, the omission of the exponent " 2 " on the r.h.s.)

Since the integrals in (5.15) can be rescaled to unit phase speed by using the similarity law (2.2), the only significant parameter in (5.15) is $s$, the separation between the two solitons. Nagashima and Kuwahara [9] have given an analytical form for $f(x)$, the soliton for $c=1$, as

$$
\begin{aligned}
f(X)= & 2.65758756 \exp \left(-0.16 X^{2}\right) \\
& \times\left[1.000794208-0.006761592432 X^{2}\right. \\
& -0.001355732644 X^{4}+2.520234609 \\
& \times 10^{-5} X^{6} \\
& \left.-4.782592684 \times 10^{-6} X^{8}\right] .
\end{aligned}
$$

Using (5.16) and (2.2) in (5.15) gives, replacing $\tau$ by $t$,

$\left[c_{1}\right]_{t}=-0.02682\left[c_{1}\right]^{9 / 4} I(S)$, 
where

$S \equiv c^{1 / 4} s$,

$I(S) \equiv \int_{-\infty}^{\infty} f^{2}(X)[f(X-S)]_{S} \mathrm{~d} X$.

We have suppressed the subscript on $c$ in (5.18) because the analysis may be repeated for the other soliton to obtain an equation identical to (5.17) for $\left[c_{1}\right]_{t}$ except for an overall minus sign. The magnitude of $c$, which is what appears in the definition of the rescaled separation $S$, is the same for both solitons (to lowest order!), but their time rate of change is equal and opposite. The minus sign in (5.10) implies (if we adopt the obvious convention of positive separation $s$ ) that the " 2 " soliton is leading the " 1 " soliton, so (5.17) describes the deacceleration of the trailing soliton.

Since $s_{t}=c_{2}-c_{1}$, it follows by differentiation with respect to $t$ that

$s_{t t}=0.05364 c^{9 / 4} I\left(c^{1 / 4} s\right)$,

which can be put in the form of Newton's equation in mechanics with a potential energy $V(s)$ by writing

$s_{t t}=-\mathrm{d} V(s) / \mathrm{d} s$,

where the potential energy function is

$$
V(s) \equiv 0.05364 c^{2} \int_{-\infty}^{\infty} f^{2}(X) f\left(X-c^{1 / 4} s\right) \mathrm{d} X .
$$

Since the time variations in the phase speeds are assumed to be $\mathcal{O}(\epsilon)$ in comparison to the phase speeds, we can take $c$ to be constant and equal to the average of the velocities of the two solitons when they are infinitely far apart. Gorshkov, Ostrovskii, and Papko [1] derive an equation similar to (5.22) except that they use an asymptotic approximation to $f\left(X-c^{1 / 4} s\right)$ to obtain an analytic approximation to $V(s)$; we numerically integrated (5.22) without approximation to avoid any error except that which is intrinsic to the

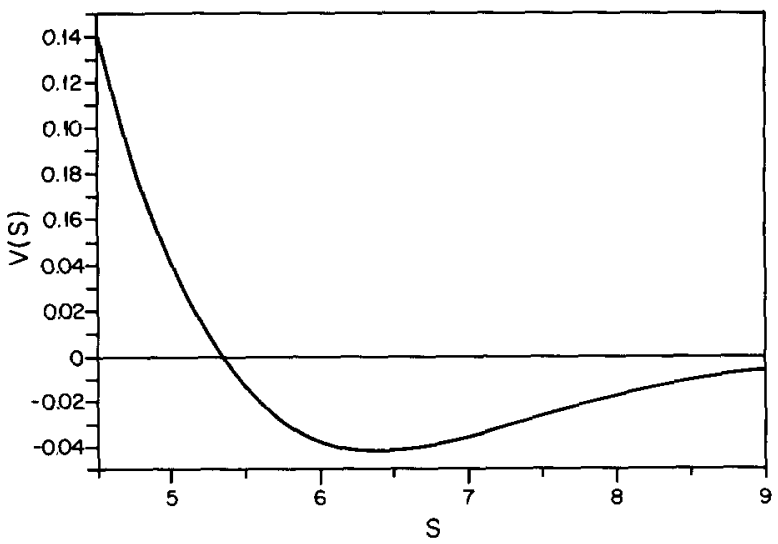

Fig. 6. The potential energy $V(s)$ for the interaction of two nearly-identical solitons in the Gorshkov-Ostrovskii-Papko theory. Here, $s$ is the separation distance between the two peaks and the mathematical form of $V(s)$ is given by (5.22). The graph is for unit phase speed; $V(s)$ for general $c$ can be obtained merely by rescaling the axes of this figure.

singular perturbation theory. Fig. 6 shows $V(s)$ for $c=1$; the potential energy for all other $c$ is of the same shape and can be obtained by rescaling according to (5.22).

The most striking feature of Fig. 5 is the large energy minimum centered at a soliton separation of $s=6.3895$ (for $c=1$ ). What this implies is that the ineraction between the two solitons is attractive rather than repulsive when the distance between them is greater than 5.33. An initial condition of two identical solitons separated by $s>$ 5.33 (where the potential energy dips below its asymptotic $(s \rightarrow \infty)$ value) will generate a bound state of solitons in which the two peaks oscillate about the minimum in the potential energy. Many soliton equations, including some that can be solved via inverse scattering methods like the sine-Gordon equation, have such oscillating bound states, which are usually called "breathers". FKDV breathers have been previously seen by Nagashima and Kuwahara [12] and Yoshimura and Watanabe [13] in numerical integrations and by Gorshkov, Ostrovskii, and Papko [1] in experiments with electrical circuits.

When the separation between the two solitons is exactly $s=6.3895$, the perturbation theory pre- 


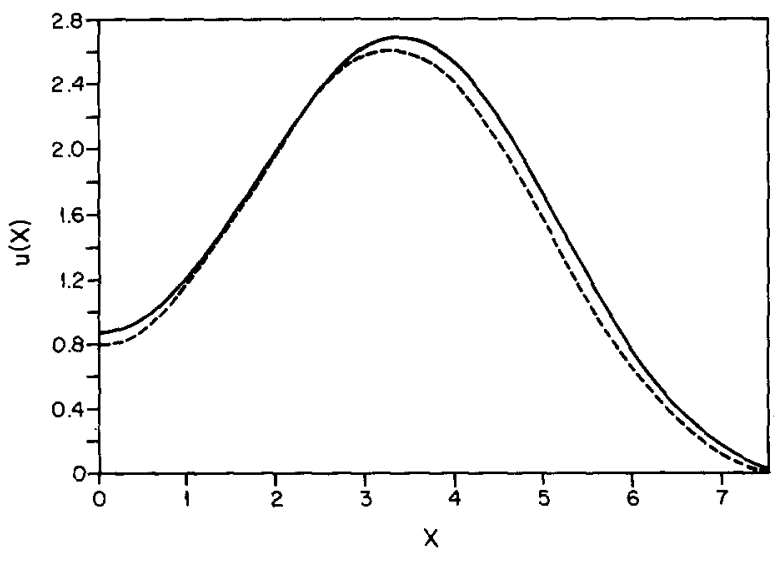

Fig. 7. The numerically computed bion (steadily-translating, double-peaked solitary wave) [solid line] is compared with the prediction of the Gorshkov-Ostrovskii-Papko theory [dashed curve], which is the sum of two ordinary solitons separated by the distance $s$ which gives the minimum of the potential energy function graphed in fig. 6 .

dicts that the two solitons will neither repel nor attract, but will be frozen at that position forever. Since this particular bound state of solitons does not "breathe" (i.e. oscillate), we will refer to it as the bion. This and its spatially period generalization, the "bicnoidal" wave, were computed by the same numerical methods as for the ordinary cnoidal wave.

Fig. 7 compares the exact bion with the superposition of two solitons separated by $s=$ 6.3895. The agreement is very good: a ringing endorsement for perturbation theory. The actual separation of the two peaks of the bion is 6.654 , a difference of only $4 \%$. The maximum of the absolute error of $u(X)$ is less than $6 \%$ of the maximum of the bion.

It must be admitted that the perturbation theory would not do as well for soliton collisions. There is no scattering for the stationary bion, and since the velocities of its two components are exactly equal and independent of time, there is no error in replacing $c_{1}(t)$ and $c_{2}(t)$ by their asymptotic average, either. However, Gorshkov, Ostrovskii, and Papko [1] show that their method gives the exact phase shift for the collision of two solitons of the Korteweg-deVries equation, so it is
Table III

An analytical expression for the bion (double-peaked, steadily translating solitary wave) of the FKDV equation. The approximation, with $c$ being the phase speed in the "soliton" convention, is

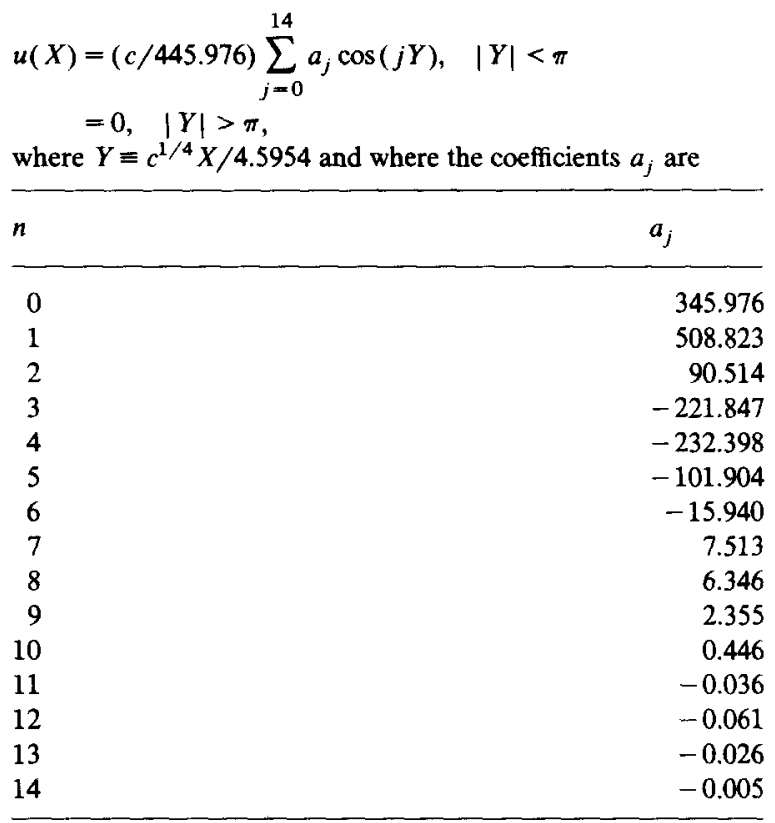

clear that their theory is useful for time-dependent soliton interactions as well.

The same authors point out that the formation of bound states is possible only when the solitons oscillate as $|X| \rightarrow \infty$. This is obvious from (5.22): the integral that defines the potential energy is positive definite unless the soliton profile $f(X)$ changes sign. As $|X| \rightarrow \infty$, the asymptotic decay of the soliton reduces the FKDV equation to the linear equation $-u_{X X X X X}-c u_{X}=0$, which implies $u(X) \approx M \exp \left(-0.707 c^{1 / 4}|X|\right)$ $\sin \left(0.707 c^{1 / 4}|X|+\phi\right)$ for some constants $M$ and $\phi$. This further implies that $V(s)$ is oscillatory and has an infinite number of potential valleys. Fig. 7 shows, however, that only the first minimum is significant; in a real physical system, it would be extremely difficult to observe bions whose equilibrium separation was determined by the second or higher troughs in $V(s)$. Consequently, only the bion associated with the first minimum is computed here. 


\section{Bifurcation: branching of the bicnoidal wave from the cnoidal wave}

Each Newton's iteration requires solving an inhomogeneous linear differential equation of the form

$$
-\Delta_{X X X X X}-c \Delta_{X}+(U[X] \Delta)_{X}=F(X),
$$

where $U(X)$ is the result of the previous iterate and $\Delta(X)$ is the correction. In discretized form, this becomes the linear matrix equation $A d=f$ as in (4.11) and the solution can be formally written in terms of the eigenvectors of the matrix $A$ as

$d=\sum_{j=1}^{N}\left[F_{j} / \lambda_{j}\right] e_{j}$,

where $A e_{j}=\lambda_{j} e_{j}$ with $F_{j}=\left(f, e_{j}\right)$ where the parentheses denotes the usual matrix inner product.

The eigenvector decomposition (6.2) shows that the iteration equation is always soluble unless one of the eigenvalues is equal to 0 , which happens at both bifurcation points and limit points. As a convention and without loss of generality, we will number the eigenvectors so that the one which goes to 0 at the singular point is $j=1$. If $F_{1} \neq 0$, then the singular point is a limit point since (6.2) shows that even slight changes in $F(X)$-equivalently, slight changes in $c$-produces enormous changes in the correction $\Delta(X)$ when $\lambda_{j} \ll 1$. At the singular point itself, $\mathrm{d} a / \mathrm{d} c=\infty$.

On the other hand, if $F_{1}=0$, then neither $\mathrm{d} a / \mathrm{d} c$ nor any of the derivatives of Fourier coefficients with respect to the parameter $c$ are forced to blow up. The solution can be smoothly continued right through the singular point. The rub is that the existence of an eigenfunction with zero eigenvalue implies that this smooth solution is no longer unique: we can add an arbitrary multiple of $e_{1}$ to the particular solution given by (6.2), and (6.1) is still satisfied. This nonuniqueness implies that this point in parameter space where $\lambda_{1}=0$ is a bifurcation point: another independent solution branches off that given by (6.2).
The eigenfunction $e_{1}$ is important because a small multiple of $e_{1}$ can be added to the particular solution (6.2) [which is the primary branch] to obtain a good first guess for switching the continuation method onto the secondary branch. It indicates (in the $\mathrm{N}$-dimensional space of Fourier coefficients) the direction of the secondary branch when it leaves the primary branch.

The bicnoidal wave of the FKDV equation, which becomes the bion for large amplitude, is a secondary branch emerging from a bifurcation point of the ordinary cnoidal wave. The bifurcation is subharmonic, which is a fancy way of saying that the bicnoidal wave has a spatial period which is twice that of the cnoidal wave from which it branches. The bicnoidal wave has two peaks on each spatial period, so it can only branch from an ordinary cnoidal wave which also has two peaks on $X \in[-\pi, \pi]$.

If we choose the spatial period of the bion to be $2 \pi$, then it branches from the " $n=2$ " cnoidal wave discussed in section 2 . This is simply the cnoidal wave of period of $2 \pi$ rescaled so as to have a period of $\pi$. This period- $\pi$ cnoidal wave then has $c=-16$ in the limit $a \rightarrow 0$, and $a$ for the cnoidal wave must now be reinterpreted as the second Fourier coefficient $a_{2}$ since the Fourier expansion of the cnoidal wave of period $\pi$ involves only $\cos (2 X), \cos (4 X), \cos (6 X), \ldots$

Fig. 8 shows both the cnoidal wave of period $\pi$ and its linear eigenfunction of zero eigenvalue at the bifurcation point $a_{2}=-39.37$. (Reversing the sign of $a_{2}$ shifts the peaks of $u(X)$ by $\pi / 2$, but is otherwise irrelevant.) When the arbitrary multiplier of the eigenfunction $e_{1}(X)$ is chosen so that $a_{1}>0$, adding $e_{1}(x)$ to the cnoidal wave shifts the peaks at $X= \pm \pi / 2$ towards the origin. Simultaneously, the trough at the origin is partly filled in while those at $X= \pm \pi$ become deeper. These trends continue when we follow the bicnoidal wave away from the bifurcation: for large amplitude, the bicnoidal wave becomes the bion - two tall, narrow peaks close to the origin. The filling-in of the trough at $X=0$ is consistent with the overlap of the bion's two peaks. 


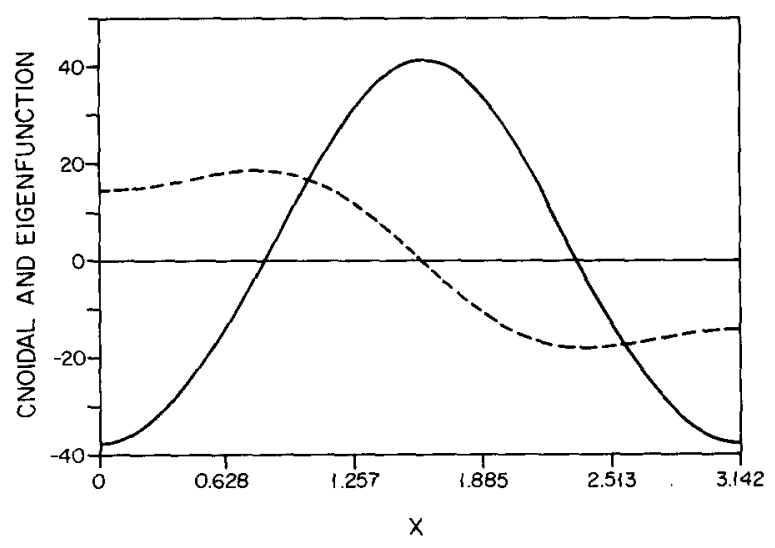

Fig. 8. The solid curve shows the cnoidal wave (of period $\pi$ ) at that amplitude where the bicnoidal wave bifurcates from it. The dashed line is the eigenfunction of zero eigenvalue of the linearized FKDV equation, which shows how the bicnoidal wave differs from the cnoidal in the neighborhood of the bifurcation point.

It is possible to combine a running check for bifurcation with the continuation method at little extra cost. The method is to replace the column vector $f$ in (4.11) by an arbitrary vector $r$ such that $\left(r, e_{1}\right) \neq 0$ and then solve for a column vector we will denote $d_{r}$. Then $\left\|d_{r}\right\| /\|r\| \rightarrow \infty$ as we approach the bifurcation. This step is cheap because we already must factorize the pseudospectral matrix in order to compute the correction to $u(X)$, so computing $d_{r}$ requires only a backsolve. This is equivalent to calculating an eigenfunction of zero eigenvalue by inverse iteration [27]. Near the bifurcation, $d_{r}$ will be proportional to $e_{1}$. This in turn allows us to switch onto the secondary branch by adding a small múltiple of this eigenfunction to $u(X)$ on the primary branch.

\section{7. "Imbricate" series and the cnoidal wave}

As noted in the introduction, a simple-minded approximation for the large amplitude cnoidal wave is a series of identical solitons spaced a distance of $2 \pi$ apart. For large amplitude, the peaks of the cnoidal wave are narrow and decay exponentially, so the overlap of adjacent peaks is small. Since the solitons are exact solutions of the equation, it follows that such a series approximates the cnoidal wave with an exponentially small error proportional to the soliton-soliton overlap.

This much is obvious. What is not so obvious is that any Fourier series can in fact be represented by a series of this same form for some appropriate pattern function $A(x)$, regardless of whether the Fourier series has any connection with nonlinear waves or not. Such series are discussed in [17] where they are named "imbricate" series after an obsolete adjective that means "to overlap like a pattern of tiles". The basic result is the following.

Theorem. Let

$u(X) \equiv \sum_{n=-\infty}^{\infty} a(n) \mathrm{e}^{\mathrm{i} n X}$

Then $u(X)$ also has the exact "imbricate" series representation

$u(X)=(2 \pi)^{1 / 2} \sum_{m=-\infty}^{\infty} A(X-2 \pi m)$,

where the "pattern" function $A(X)$ is the Fourier transform of the function $a(n)$ that gives the coefficients of the ordinary Fourier series:

$A(X)=(2 \pi)^{-1 / 2} \int_{-\infty}^{\infty} a(n) \mathrm{e}^{\mathrm{i} n X} \mathrm{~d} n$.

The proof [17] is a trivial application of the Poisson summation formula. The imbricate series for a given $u(X)$ is not unique because there exists an infinity of continuous functions $a(n)$ which interpolate to the coefficients of the Fourier series. Knowledge of the pattern function $A(X)$, however, does uniquely determine the coefficients of the Fourier series and $u(X)$.

For cnoidal waves, the obvious zeroth order approximation is to choose the pattern function $A(x)$ to be the isolated soliton on the interval $X \in[-\infty, \infty]$. In 1975, however, M. Toda [19] proved the remarkable theorem - undiscovered in 
the 80 years since the cnoidal wave solution was first given in 1895 - that the "imbricate" series of evenly spaced solitons is an exact solution for the Korteweg-deVries equation for all amplitudes, even when the cnoidal wave differs little from a cosine wave and the soliton peaks are much wider than the period of the wave. (The phase speed, however, is not exactly equal to that of the solitons in the series and must be calculated as in [21].)

Boyd [29] has shown via the Poisson summation formula that Toda's theorem can be extended to other combinations of elliptic functions and therefore to cnoidal waves of virtually all equations that are "exactly integrable", i.e. belong to that very special class which is solvable through the inverse scattering transform. The FKDV equation is not exactly integrable and its cnoidal wave cannot be expressed in terms of elliptic functions except for a single isolated parameter value. Nonetheless, it is impossible to furnish the reader with a useful graph comparing the imbricate soliton series with the exact cnoidal wave because the difference is less than the thickness of the curve - less than 0.005 of the maximum of $|u(X)|$-for all amplitudes. Table IV compares the cnoidal wave with its imbricate-soliton approximation for various values of $a$. The phase speed formula, as true even for the Korteweg-deVries equation, is only an approximation, one that deteriorates (albeit non-monotonically) as $a \mathrm{de}$ creases and the solitons overlap more and more.

The accuracy of the representation of the spatial structure is astonishingly good, however. An amplitude of $a=1$ is very small, very deep in the sine wave regime: the cnoidal wave is approximated to within $1.7 \%$ (relative to $\max |u(X)|$ ) by a single cosine. Fig. 9 shows the cnoidal wave for $a=1$ with the three solitons of the imbricate series which are important for $X \in[0, \pi]$. (This graph is presented in the "soliton" convention, i.e. the constant $a_{0}=1.81$ has been added to the cosine terms, because this constant cannot be subtracted from individual solitons but only from the imbricate series as a whole). At the origin, the soliton
Table IV

A comparison of the Fourier coefficients of the exact cnoidal wave with those of the imbricate-soliton series approximation (7.2) for several amplitudes. The approximate phase speeds (which are those of the solitons in the imbricate series) and the maximum point-wise error are also given. All results are in the "cnoidal" convention and are normalized so that the coefficient of $\cos (X)$ is the same for both exact cnoidal wave and approximation, which can always be done by appropriately varying the width of the solitons in the imbricate series.

\begin{tabular}{|c|c|c|c|c|}
\hline$n$ & $\begin{array}{l}\text { Exact } c \\
\text { or } a_{n}\end{array}$ & $\begin{array}{c}\text { Approximate } c \\
\text { or } a_{n}\end{array}$ & Absolute err. & Relative err. \\
\hline \multicolumn{5}{|c|}{$a=50$} \\
\hline$c$ & 14.683 & 14.631 & 0.05 & $0.4 \%$ \\
\hline 1 & 50. & 50. & - & - \\
\hline 2 & 27.101 & 27.220 & 0.120 & $0.4 \%$ \\
\hline 3 & 7.476 & 7.473 & -0.003 & $0.04 \%$ \\
\hline 4 & 1.390 & 1.371 & -0.019 & $1.4 \%$ \\
\hline 5 & 0.214 & 0.205 & -0.009 & $4.3 \%$ \\
\hline 6 & 0.029 & wrong sign & $* * * * * *$ & $* * * *$ \\
\hline \multicolumn{5}{|c|}{$\operatorname{Max}\left|u(X)-u_{\text {imbricate }}(X)\right|<0.160$ for all $X$} \\
\hline \multicolumn{5}{|c|}{$\underline{a=10}$} \\
\hline$c$ & -0.1856 & -0.2481 & 0.06 & $33.6 \%$ \\
\hline 1 & 10. & 10 & - & - \\
\hline 2 & 1.613 & 1.574 & -0.039 & $2.4 \%$ \\
\hline 3 & 0.100 & 0.105 & 0.005 & $4.5 \%$ \\
\hline 4 & 0.005 & wrong sign & $* * * * *$ & $* * * * *$ \\
\hline \multicolumn{5}{|c|}{$\operatorname{Max}\left|u(X)-u_{\text {imbricate }}(X)\right|<0.055$ for all $X$} \\
\hline \multicolumn{5}{|c|}{$\underline{a=5}$} \\
\hline$c$ & -0.7930 & -0.8146 & 0.022 & $2.7 \%$ \\
\hline 1 & 5. & 5. & - & - \\
\hline 2 & 0.413 & 0.374 & -0.039 & $9.4 \%$ \\
\hline 3 & 0.013 & 0.004 & -0.009 & $70.4 \%$ \\
\hline 4 & 0.0003 & wrong sign & $* * * * * *$ & $* * * * *$ \\
\hline \multicolumn{5}{|c|}{$\operatorname{Max}\left|u(X)-u_{\text {imbricate }}(X)\right|<0.050$ for all $X$} \\
\hline \multicolumn{5}{|c|}{$\underline{a=1}$} \\
\hline$c$ & -0.9916 & -0.7214 & -0.270 & $27.3 \%$ \\
\hline 1 & 1. & 1. & - & - \\
\hline 2 & 0.0166 & 0.0134 & -0.0033 & $19.9 \%$ \\
\hline 3 & 0.0001 & wrong sign & $* * * *$ & $* * * * *$ \\
\hline & $x \mid u(X)-\imath$ & nbricate $(X) \mid<0$ & 004 for all $X$ & \\
\hline
\end{tabular}

centered on $X=0$ is taller than the cnoidal wave by 0.104 -but this is cancelled in the imbricate series by the solitons centered on $X= \pm 2 \pi$, whose 


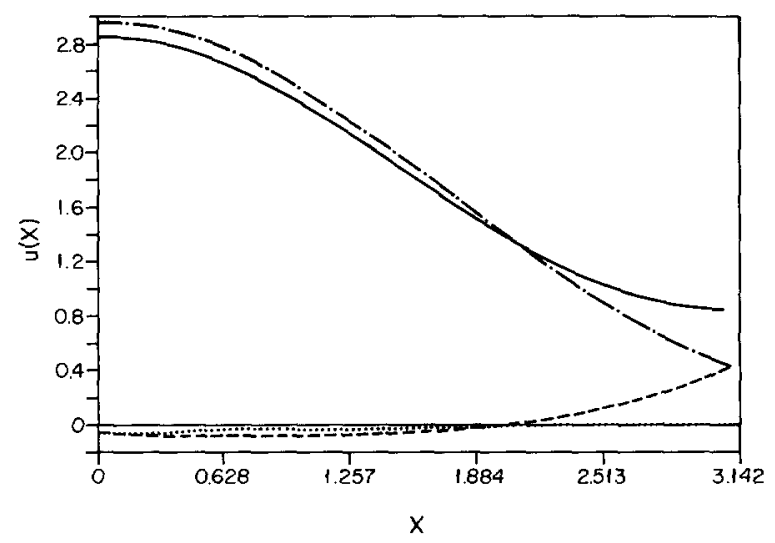

Fig. 9. The cnoidal wave for $a=1$ (converted to the soliton convention) is the solid line; the dot-dash, dashed, and dotted curves are solitons centered on $X=0,2 \pi$, and $-2 \pi$. The imbricate-soliton series which is the superposition of these solitons approximates the cnoidal wave to within the thickness of the curve even though this small amplitude wave differs negligibly from the sum of a constant plus $\cos (X)$.

values at $X=0$ are both -0.054 . At $X=\pi$, the soliton at $X=-2 \pi$ has decayed to a very small value, but the solitons at the origin and at $X=2 \pi$ both evaluate to 0.422 ; adding them gives a good approximation to the value of the cnoidal wave, 0.847 . The manner in which the various solitons constructively or destructively interfere to produce the sum of the imbricate series thus varies dramatically from point to point, but the end result is the same everywhere: an approximation which never differs from the exact $u(X)$ by more than 0.004 over the whole interval - even though this cnoidal wave is essentially a cosine plus a constant.

The remarkable degree to which Toda's theorem is approximately satisfied even for this non-integrable equation cries out for an explanation, but we can only offer heuristic comments. One is to invoke the theorem stated above: every periodic function has an imbricate series, so the mystery has been reduced to explaining why the pattern function of that series is so close to the soliton even for small amplitude.
The second point is furnished by fig. 9. The pattern function of the cnoidal wave must be the soliton for large amplitude because in that case the peaks of the cnoidal wave are narrow and non-overlapping so that each peak - each repetition of the pattern function - must be an isolated nonlinear solution, i.e. a soliton. The degree to which the pattern function deviates from the soliton as the amplitude is reduced will depend on the extent to which the solitons overlap. Fig. 9 shows that, just as is true for the Korteweg-deVries equation (see figs. 2 and 3 of Boyd [21]), the solitons of the imbricate series overlap rather weakly even for $a=1$. At $X=\pi$, where the overlap is largest, the amplitude of the two solitons is each only $14 \%$ of peak amplitude, which implies that the soliton-soliton interaction terms are uniformly small in comparison to the linear terms and the self-interaction of each soliton. Table IV shows that the result of this weak overlap is that the imbricate series gives a good approximation not only to the coefficient of $\cos (X)$, but to that of $\cos (2 X)$ as well.

The conclusion is obvious: if one knows the soliton solutions to a differential equation, the imbricate series formed from them is likely to be a very powerful and useful approximation for understanding the corresponding cnoidal waves.

\section{Summary and prospectus}

The inverse scattering method is a very powerful tool for that small class of equations for which it works, but its very success may have contributed to an unfortunate neglect of the far larger class of nonlinear equations which are "non-integrable", and of algorithms to solve them. The central theme of this work is that there are rather large numbers of analytical and numerical tools for "non-integrable" problems like the fifthdegree Korteweg-deVries equation. 
We have obtained new results for the FKDV including: (i) Stokes' expansions and Padé approximants for the cnoidal wave; (ii) numerical calculations of both the cnoidal and bicnoidal waves for a wide range of amplitudes; (iii) an analytical expression for the bion; (iv) calculation of the bifurcation point where the bicnoidal wave branches from the cnoidal; (v) an explicit test of the Gorshkov-Ostrovskii-Papko perturbation theory by comparing the predicted bound state of solitons with the exact bion; and (vi) a numerical verification of the high accuracy of the imbricatesoliton series for the spatial structure of the cnoidal wave even for extremely small amplitudes. These will provide a good background for further work on the FKDV equation.

A larger question, however, is: can the methods of this paper be applied in two or three dimensions? Models with but a single dimension are useful for conceptual purposes, but the world is not really made that way. Some ideas, like that of the imbricate-soliton approximation to the cnoidal wave and the Gorshkov-Ostrovskii-Papko theory of interacting solitons, generalize to higher dimensions in a trivial way. The cost of the Stokes' expansion increases by $\mathcal{O}(N)$ in two dimensions and $\mathcal{O}\left(N^{2}\right)$ in three dimensions over the onedimensional case where $N$ is the order of the series, but the one-dimensional calculation was so cheap - 3 minutes in interpreted BASIC! - that this method can be easily applied even in three dimensions.

The Newton/pseudospectral/continuation polyalgorithm, alas, is both the most powerful and robust method and also the most expensive: if we use $N$ degrees of freedom in each dimension, then we have to invert an $N^{d} \times N^{d}$ matrix at each step where $d$ is the dimension. Since we performed most calculations with $N \leq 10$, however, it is obvious that many two-dimensional problems can be computed quite inexpensively.

Overall, the outlook is optimistic. The example of the FKDV equation has shown how much can be done to understand solitary and cnoidal waves even for "non-integrable" equations. Work in progress will apply these ideas to more realistic twodimensional problems.

\section{Acknowledgements}

This work was supported by the National Science Foundation under grants OCE8305648 and OCE8509923.

\section{References}

[1] K.A. Gorshkov, L.A. Ostrovskii and V.V. Papko, Sov. Phys. JETP 44 (1976) 306.

[2] K.A. Gorshkov, L.A. Ostrovskii and V.V. Papko, Sov. Phys. Dokl. 22 (1977) 378

[3] K.A. Gorshov and V.V. Papko, Sov. Phys. JETP 46 (1977) 92.

[4] K.A. Gorshkov, L.A. Ostrovskii, V.V. Papko and A.S Pikovsky, Phys. Lett. 74A (1979) 177.

[5] K.A. Gorshkov and L.A. Ostrovskii, Physica 3D (1981) 428.

[6] M. Imada, J. Phys. Soc. Japan 52 (1983) 1946.

[7] K. Kano and T. Nakayama, J. Phys. Soc. Japan 50 (1981) 361

[8] T. Kawahara, J. Phys. Soc. Japan 33 (1972) 260.

[9] H. Nagashima and M. Kuwahara, J. Phys. Soc. Japan 50 (1981) 3792

[10] A. Nayfeh, Perturbation Methods (Wiley, New York, 1973) p. 58.

[11] L.A. Ostrovskii and E.N. Pelinovskii, Sov. Phys. Dokl. 15 (1971) 1097

[12] Y. Yamamoto and E.I. Takizawa, J. Phys. Soc. Japan (1981) 1421

[13] K. Yoshimura and S. Watanabe, J. Phys. Soc. Japan 51 (1982) 3028

[14] C.M. Bender and S.A. Orszag, Advanced Mathematical Methods for Scientists and Engineers (McGraw-Hill, New York, 1978) p. 383.

[15] J.P. Boyd, Mon. Wea. Rev. 106 (1978) 1192.

[16] D. Gottlieb and S.A. Orszag, Numerical Analysis of Spectral Methods (SIAM, Philadelphia, 1977).

[17] J.P. Boyd, J. Comp. Phys., to be published.

[18] T.F. Chan and H.B. Keller, SIAM J. Sci. Stat. Comput. 3 (1982) 173.

[19] M. Toda, Phys. Rept. 18 (1975) 1.

[20] S. Orszag, J. Comp. Phys. 37 (1980) 70.

[21] J.P. Boyd, J. Math. Phys. 23 (1982) 375.

[22] D.W. McLaughlin and A.C. Scott, Phys. Rev. A18 (1978) 1652. 
[23] J.P. Keener and D.W. McLaughlin, Phys. Rev. A16 (1977) 777.

[24] H.J. Norton, Comp. J. 7 (1964) 76.

[25] L.V. Kantorovich, Dokl. Akad. Nauk SSSR 59 (1948) 1237.

[26] P. Lax, Commun. Pure Appl. Math. 21 (1968) 467.
[27] D.M. Young and R.T. Gregory, A Survey of Numerical Mathematics, vol. 2 (Addison-Wesley, Reading, MA, 1973) p. 918

[28] B.A. Finlayson, The Method of Weighted Residuals and Variational Principles (Academic Press, New York, 1972).

[29] J.P. Boyd, SIAM J. Appl. Math. 44 (1984) 952. 